A review of the genetics of Dravet syndrome (severe myoclonic epilepsy of infancy, SMEI) finds a genetic etiology and SCN1A mutations in $70 \%-80 \%$ of patients; in $20 \%$ the cause is unknown. Are $S C N 1 A$ gene abnormalities essential for the diagnosis of Dravet syndrome, or are other genes sometimes involved? (Marini C et al. Epilepsia 2011;52 (Suppl 2):24-29). Five alleged cases of pertussis vaccine encephalopathy were rediagnosed years later as Dravet syndrome, testing positive for SCN1A mutations. (Reyes IS et al. Pediatrics 2011;128(3):e699-e702). More frequent SCN1A genetic testing in infants with refractory myoclonic seizures should lead to earlier diagnosis and more effective treatment of Dravet syndrome cases.

\title{
COPY NUMBER VARIANTS IN EPILEPTIC ENCEPHALOPATHY
}

An international group of investigators at University of Washington, Seattle, USA, and various centers in Australia, New Zealand, Canada, and Israel evaluated 315 patients with epileptic encephalopathies for rare copy number variants (CNVs) using a whole-genome oligonucleotide array. Twenty five (7.9\%) patients carried rare CNVs thought to contribute to their phenotype, one half being pathogenic. Several novel candidate genes for epilepsy were uncovered. Array comparative genomic hybridization (CGH) should be considered in the genetic evaluation of patients with epileptic encephalopathy characterized by severe epilepsy and cognitive regression. (Mefford HC, Yendle SC, Hsu C, et al. Rare copy number variants are an important cause of epileptic encephalopathies. Ann Neurol Dec 2011;70:974-985). (Respond: Dr Heather C Mefford, 1959 NE Pacific St, Box 356320, Seattle, WA. E-mail: hmefford@u.washington.edu).

COMMENT. Epileptic encephalopathies (EEs) are severe epilepsies in which the epilepsy activity contributes to cognitive impairment or regression and poor outcome. Most EEs begin in infancy or childhood, often associated with normal development initially and with subsequent cognitive decline. These cases differ from those epilepsies with static intellectual disability. Copy number variants are an important source of gene mutation in neurocognitive disorders and the epilepsies.

The gene content of copy number variants found in 11 subjects with infantile spasms was involved in abnormalities of ventral forebrain development and pathways of synaptic function (Paciorkowski AR et al. Eur J Hum Genet 2011;19(12):1238-1245).

\section{EPILEPTIC ENCEPHALOPATHIES, CDKL5 MUTATIONS, AND INFANTILE SPASMS}

Researchers at the Mayo Clinic, Rochester, $\mathrm{MN}$ performed retrospective chart reviews of 6 children with epilepsy and CDKL5 mutations. Four were girls and 2 boys. All developed infantile spasms after the majority (4/6,67\%) presented with partial-onset seizures. Five had dysphagia, profound in 4. The EEG revealed hypsarrhythmia in 3 children and modified hypsarrhythmia in 2. Mean age of seizure onset was 1.8 months (range, 1-3 months). Four had hypotonia, and all had developmental delay and cortical visual impairment. Topiramate, vigabatrin, and the ketogenic diet were of most benefit, but all had refractory seizures at follow-up. Steroids or ACTH were used in 4 patients, without complete seizure control. Boys and girls were affected equally, despite the X- 